\title{
Dye Injection Flow Visualization Around a Rotating Circular Cylinder
}

\author{
Coskun Ozalp ${ }^{* *}\left(\mathbb{D}\right.$, Cemre Polat $^{1}\left(\mathbb{D}\right.$, D. Burak Saydam ${ }^{1}\left(\mathbb{D}\right.$, Mustafa Soyler $^{1}(\mathbb{C}$ \\ 'Osmaniye Korkut Ata University, Faculty of Engineering, Department of Energy Systems Engineering, Osmaniye, Turkey
}

\begin{abstract}
In this study, the visualization of the flow structure around a circular cylinder was investigated with dye injection experiments. The experiments were carried out in the closed-circuit open water channel in Osmaniye Korkut Ata University. Experiments have been studied on a circular cylinder at different rotational speeds. Experiments were carried out at $\operatorname{Re}=1000$ according to cylinder diameter and free stream velocity. In the study, with the increase of the rotation speed, an acceleration layer occurs between the fluid velocity in the cylinder wake in the flow direction and the flow from the circumference of the cylinder and the speed of rotation. This situation caused the complete disappearance of the vortex ruptures in the flow direction and the emergence of Kelvin Helmhotz fluctuations. As a result of the study, it is seen that the flow control is insufficient at low rotational speeds, but the flow can be controllable at high rotational speeds.
\end{abstract}

Keywords: Dye injection, Visualization, Circular cylinder, Flow control

\section{INTORDUCTION}

The flow created by the rotation of the roller around its transverse axis reveals a phenomenon known as the 'Robin Magnus effect'. There are many examples of the Robin Magnus effect in real life, such as the oscillation of cricket or soccer balls, the rotation of bullets around the axis of symmetry, and the transport of particles in liquids. The interactions between the inertial force of the incoming flow and the centrifugal forces caused by rotation under the effects of viscous damping lead to complex vortex structures [1]. The investigation of the flow structure around the cylinder is the subject of many researches due to its academic appeal and its practicality in many engineering applications. In the counterclockwise rotating cylinder, when the natural operating frequency of the cylinder is higher than the separation frequency, a clockwise reactive vortex is formed that starts in the upper half of the cylinder. When clockwise rotation begins, a counterclockwise vortex is formed in the lower half, resulting in a synchronized vortex mode [2]. It creates an asymmetry in the velocity and pressure fields around the rotating cylinder, which exerts a force on the cylinder normal to the flow direction. The average lift and drag coefficients and the Strouhal number increase linearly with the rotational speed. When the rotation speed is higher than the critical value, the Kármán vortex street disappears and the flow becomes steady [3]. Flow around a rotating cylinder has prevalent uses in many different industrial appli- cations. For example, there are many different areas of use, from heat exchangers, fuel and control rods used in nuclear power plants, from the paper and textile industry to cylindrical cooling devices in the glass and plastic industry [4]. Flow structure around bluff bodies have great importance in engineering applications. For example, vortex vibrations, acoustic sound or resonances around the object may occur [5]. Flow control methods are used to reduce or eliminate these effects. Flow control provides powerful tools for engineers, scientists and product developers to achieve desired goals in both internal and external flows [6].

Basically, flow control is examined in two groups as active and passive. Active flow control allows the flow structure to be changed at any time and often to eliminate the irregularities that arise. In fact, active control methods provide flexibility in control strategies. Therefore, it is possible to see many different studies in which active flow methods are applied in the literature [7-12]. Gad-el-Hak and Bushnell [7] conducted a compilation study in which different methods including flow control with moving surface boundary layer of a rotating cylinder. Tokumaru and Dimotikes [8] applied a control technique by experimentally giving rotary oscillations to the cylinder in the wake flow region formed in the cylinder downstream region. Lam [9] experimentally investigated flow in a rotating cylinder at $3600 \leq \operatorname{Re} \leq 5000$ and $\alpha \leq 2.5$ with PIV. Although the shape of the vortex is very similar to the 
vortex path behind a fixed cylinder, it is seen that the vortex becomes increasingly narrower and tumbles sideways with increasing rotation speed of the cylinder. The researcher found that the length of the vortex formation decreases as $\alpha$ increases.

In this study, flow visualization was carried out in order to have preliminary information about the flow structure around a fixed and rotating cylinder to visualize the flow. The results obtained in this study are expected to serve as an example to experimental and numerical studies and to be a reference for future studies.

\section{EXPERIMENTAL SETUP}

The experiments were carried out in the open surface water channel in the Osmaniye Korkut Ata University Advanced Fluid Mechanics Laboratory. Figure 1 contains the technical drawing visual of the water channel. The field of view in the water channel is made of plexiglass material. Two speed-controlled pumps, two collecting pools, one honeycomb pattern and mesh equipment are used to regulate the flow. The water tunnel has a length of $15 \mathrm{~m}$, a height of $1.8 \mathrm{~m}$ and a width of $4 \mathrm{~m}$. The test section where the experiments are carried out in the water tunnel has an open top and a length of $6 \mathrm{~m}$ with a cross section of $80 \times 80 \mathrm{~cm}$. Before the flow enters the test section, the flow passes through a sedimentation tank, a honeycomb and a 2: 1 constriction.

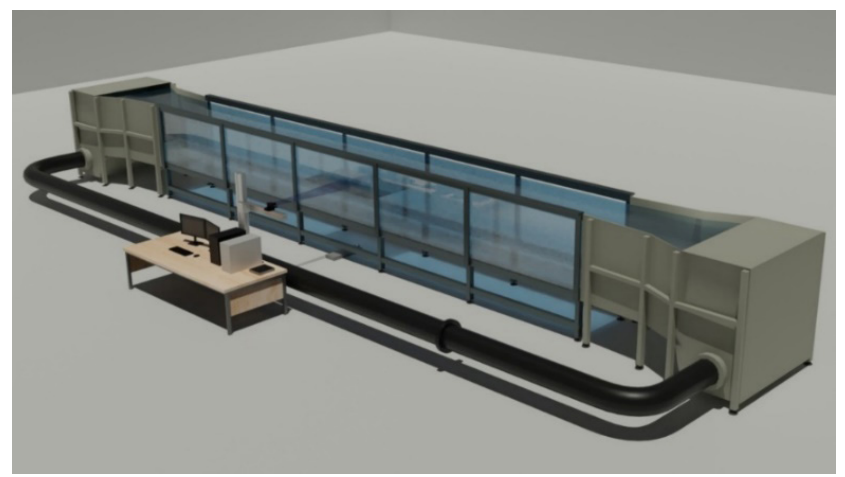

Figure 1. Technical drawing of closed-circuit open water channel

In the study, the flow field was examined by visualizing in order to have preliminary information about the flow structure. For the visualization of the flow, images of at least 66 seconds were taken with a Canon EOS M50 (24MP) model digital mirrorless camera at $1280 * 720$-pixel resolution can be capable of shooting 100 frames per second, then the images were analyzed and images representing the flow structure were obtained for each experiment. In order to obtain a non-pulsed laser beam, a horizontal laser beam was obtained using a cylindrical lens in front of the point laser and the flow was visualized. In the flow visualization, the dye / water mixture obtained by mixing Rhodamine $6 \mathrm{G}$ in powder form with water, which reflects under laser light used for detailed observation of the flow structure. During the experiments, dye was injected into the flow with a thin needle from a distance without affecting the flow from the front of the cylinder. A laser beam was sent to the channel from a certain height and images were obtained by means of the camera located under the channel (Figure 2-1). Collected images were analyzed through package programs. In addition, a servo system has been designed to give the desired rotational speed to the cylinder. The experimental setup designed for flow visualization is given in Figure 2-2. In Figure 2-2, 1- dye tank, 2-dye injector fixing plate, 3-dye injector, 4- cylinder, 5-servo motion system.
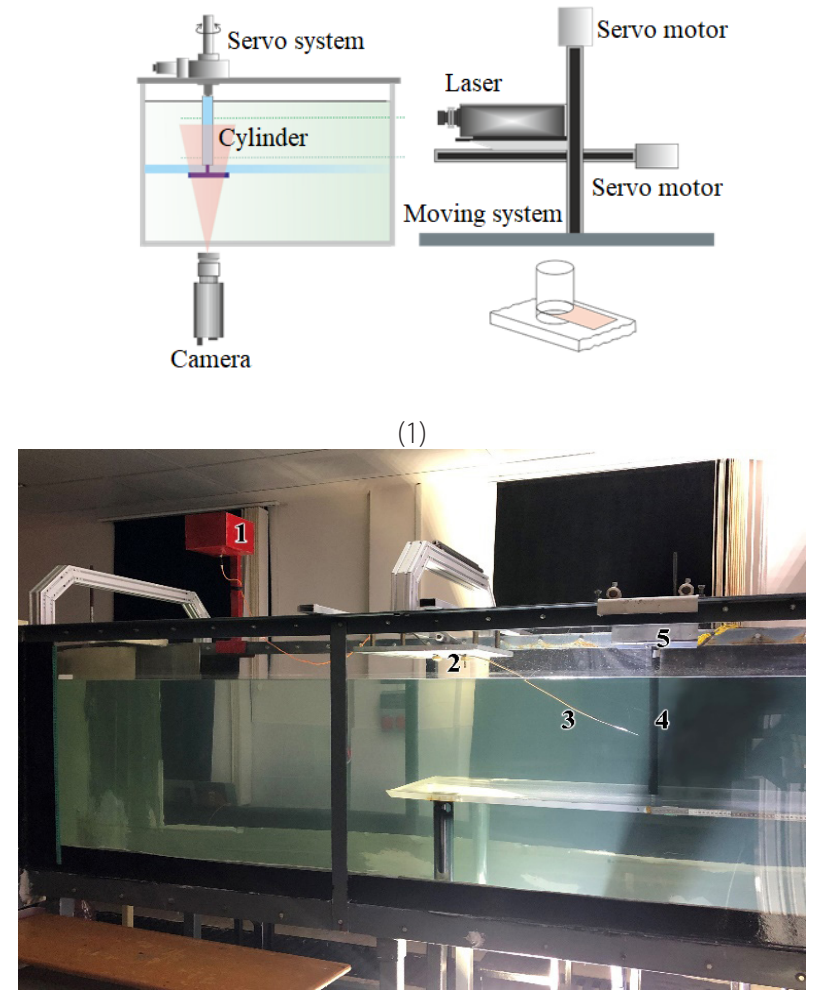

(2)

Figure 2. 1) Schematic and 2) Real image of the experimental setup

\section{RESULTS AND DISCUSSION}

In order to have preliminary information about flow characteristics around the rotating cylinder, flow visualization was carried out with dye injection experiments. In the study, the cylinder with $\mathrm{D}=25 \mathrm{~mm}$ was produced from resin material by $3 \mathrm{D}$ printing method. Different rotational speeds (rpm) and different rotation angles $(\theta)$ are given to the cylinder with servo motion system. In Figure 3-8, snapshots of the flow visualization experiments performed at $\mathrm{Re}=1000$ for the fixed cylinder are given. Reynolds number is calculated based on cylinder diameter and free stream velocity $\left(\mathrm{V}_{\mathrm{w}}\right)$. The images taken as a result of the flow visualization were created by selecting the snapshot interval at a certain time interval. In the figure, the time interval between two snapshots is selected as $0.03 \mathrm{sec}$. Dye experiments at different rotational speeds ( $\mathrm{rpm})$ were repeated for the rotating smooth cylinder, thus the effect of the rotation speed on the flow was investigated. In the figures, there are visuals taken at different time intervals in the flow visualization made in straight fixed, clockwise direction, $15 \mathrm{rpm}, 30 \mathrm{rpm}, 45 \mathrm{rpm}, 60 \mathrm{rpm}$ and $120 \mathrm{rpm}$, respectively. The formation of the Karman vortex street with a certain frequency and its progress in the di- 


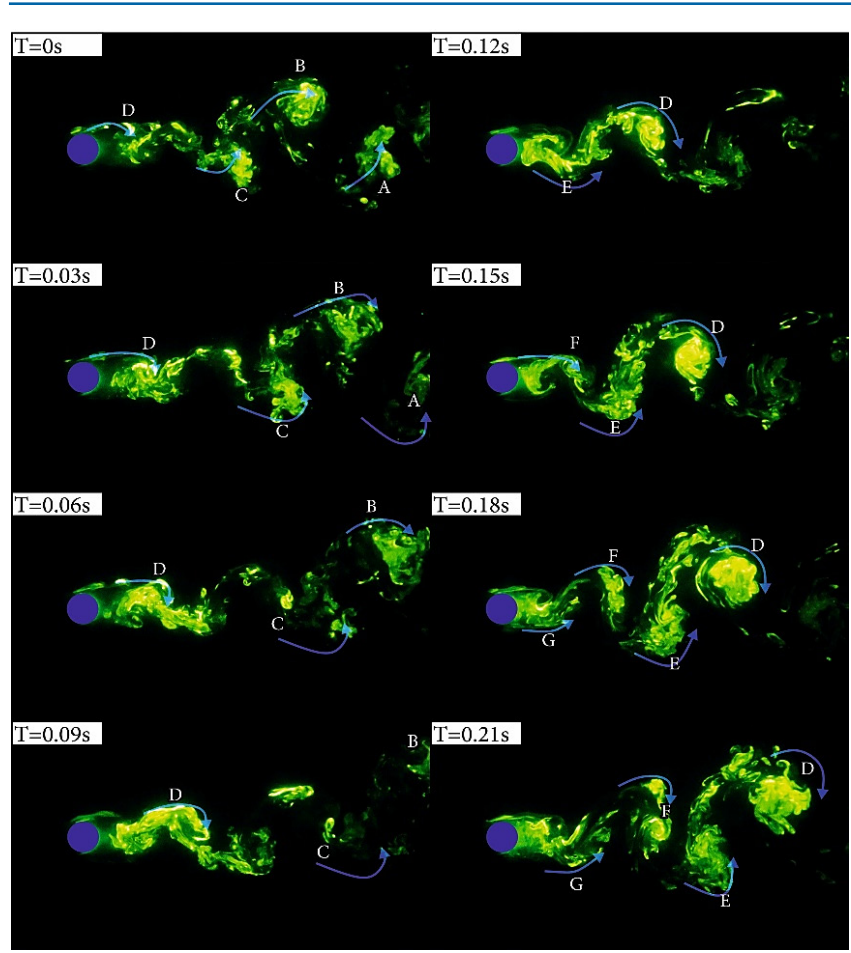

Figure 3. Flow structure formed behind fixed cylinder at $\mathrm{Re}=1000$

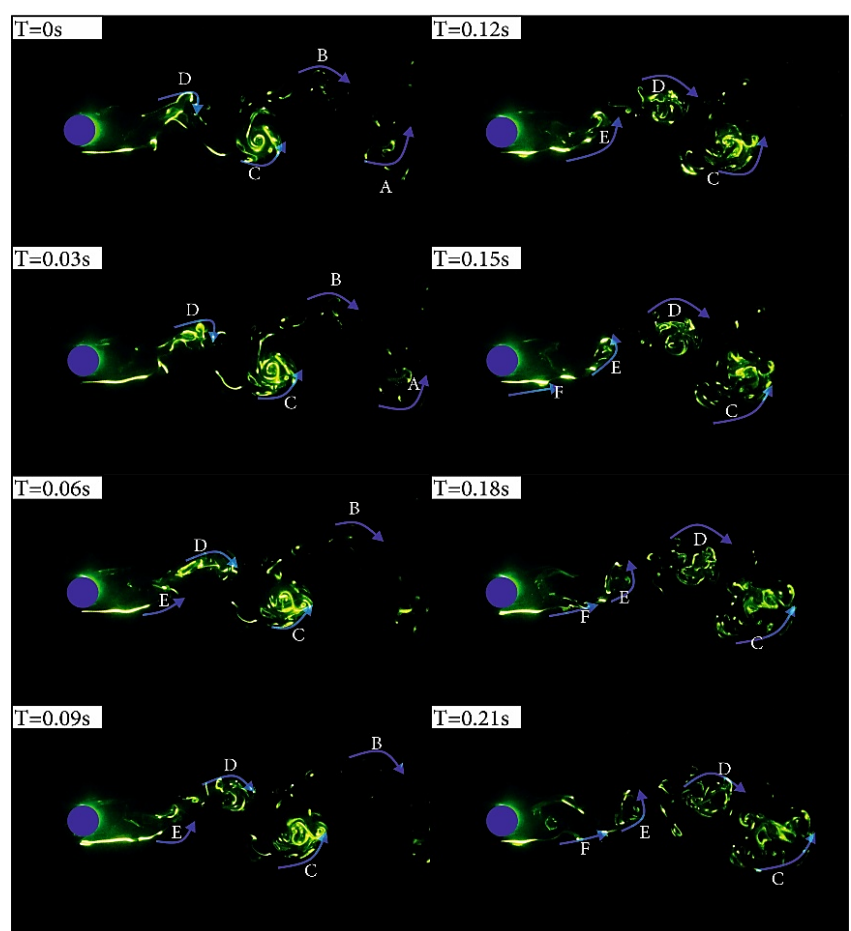

Figure 4. Flow structure formed behind the cylinder at $15 \mathrm{rpm}$ rotation clockwise at $\mathrm{Re}=1000$

rection of flow can be clearly seen from the figure (Figure 3). It is seen from the figure that the vortices (A-B-C-D) formed by separating from the cylinder boundary layer progress in time and grow (D) as they move away from the cylinder. At low rotational speeds, eddies began to form and the Karman vortex street was formed in the direction of rotation. However, the narrowing of the vortices formed by the effect of the rotation shrinks the Karman vortex street compared to the non-rotating cylinder (Figure 3-4). It has been observed that the wake region where the boundary layer separation is shifted at high rotation speeds narrows towards the cylinder and that the Karman vortex street formed in the flow di-
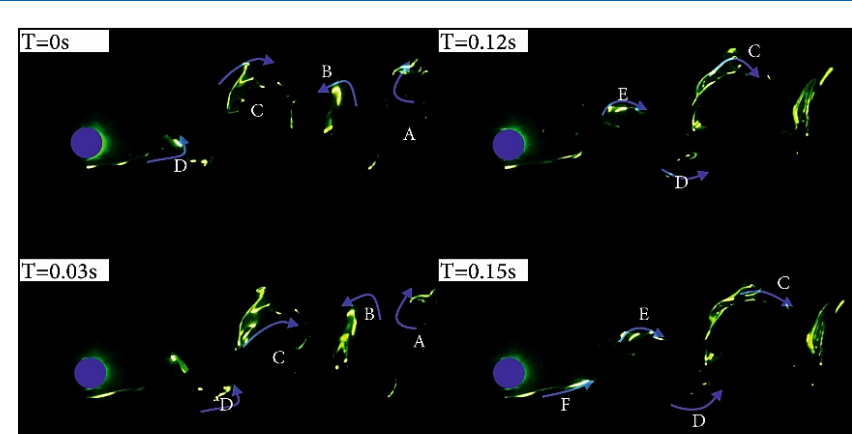

\section{$\mathrm{T}=0.06 \mathrm{~s}$}

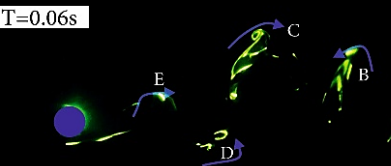

$\mathrm{T}=0.18 \mathrm{~s}$

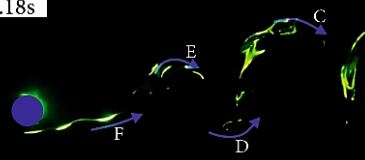

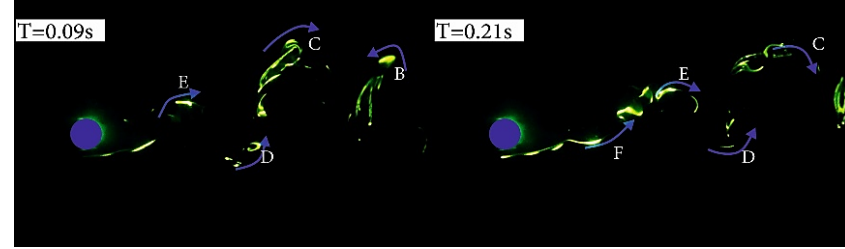

Figure 5. Flow structure formed behind the cylinder at $30 \mathrm{rpm}$ rotation clockwise at $\operatorname{Re}=1000$

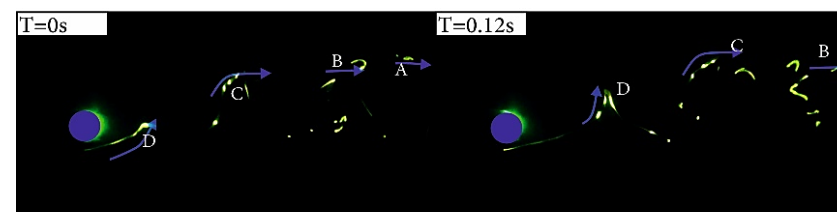

$\mathrm{T}=0.03 \mathrm{~s}$ $\vec{C} \quad \vec{B}$

$\mathrm{T}=0.15 \mathrm{~s}$

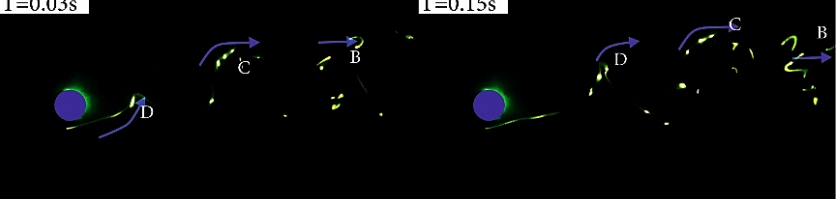

$\mathrm{T}=0.06 \mathrm{~s}$

$\mathrm{T}=0.18 \mathrm{~s}$

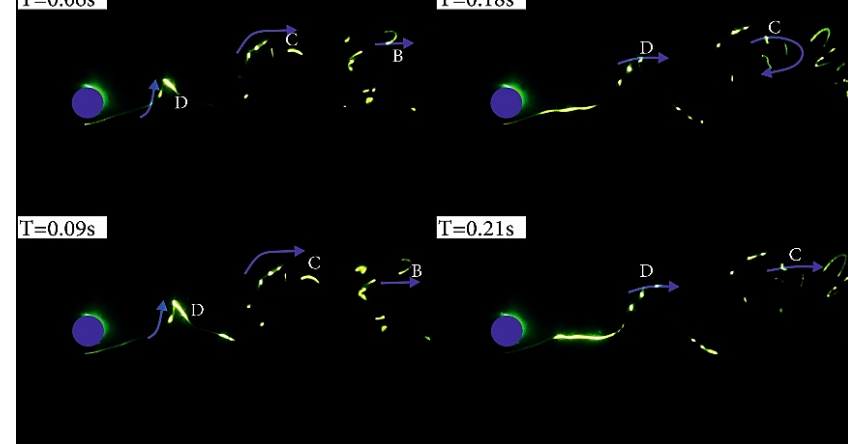

Figure 6. Flow structure formed behind the cylinder at $45 \mathrm{rpm}$ rotation clockwise at $\mathrm{Re}=1000$

rection and the instability of Kelvin Helmhotz do not occur. With the increase in the rotational speed, an acceleration layer occurs between the fluid velocity in the cylinder wake in the flow direction and the flow from the circumference of the cylinder and the increasing flow (Figure 5-8). This situation caused the vortex ruptures in the flow direction to disappear completely and the flow to proceed in the direction of rotation without distortion. When the snapshots are examined, it is seen that the flow is insufficient in controlling the flow at low cycles ( $15 \mathrm{rpm}, 30 \mathrm{rpm}$ and $45 \mathrm{rpm}$ ), but that the flow is controlled at high speeds (60 rpm and $120 \mathrm{rpm}$ ). 


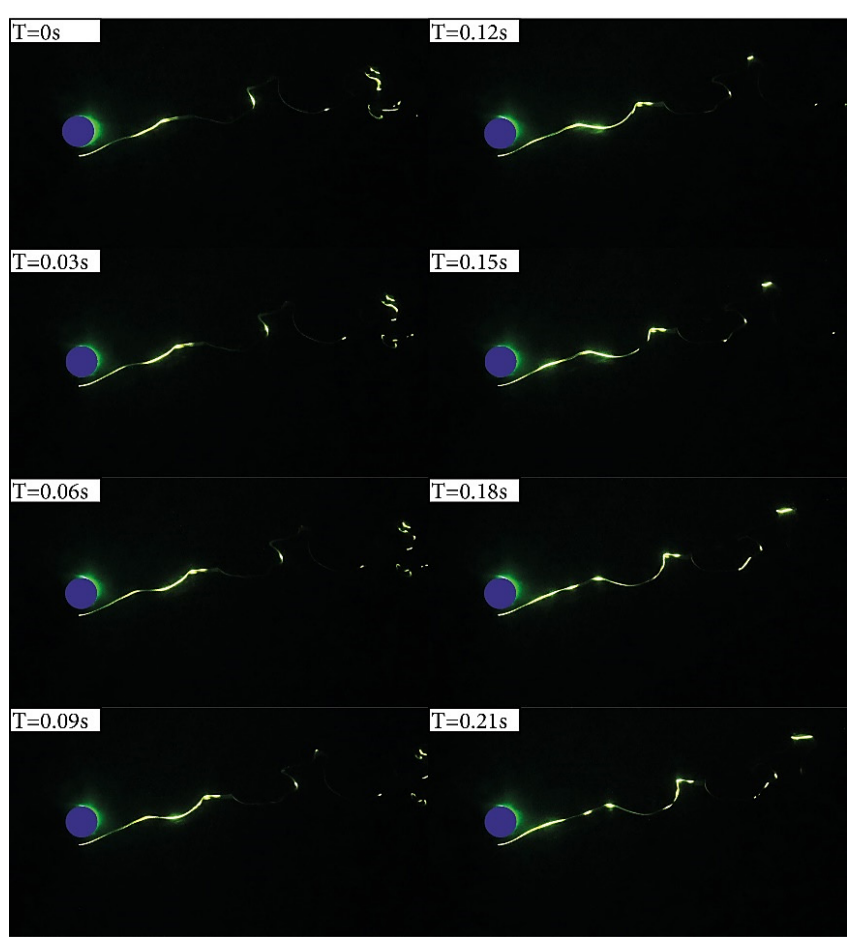

Figure 7. Flow structure formed behind the cylinder at $60 \mathrm{rpm}$ rotation clockwise at $\mathrm{Re}=1000$

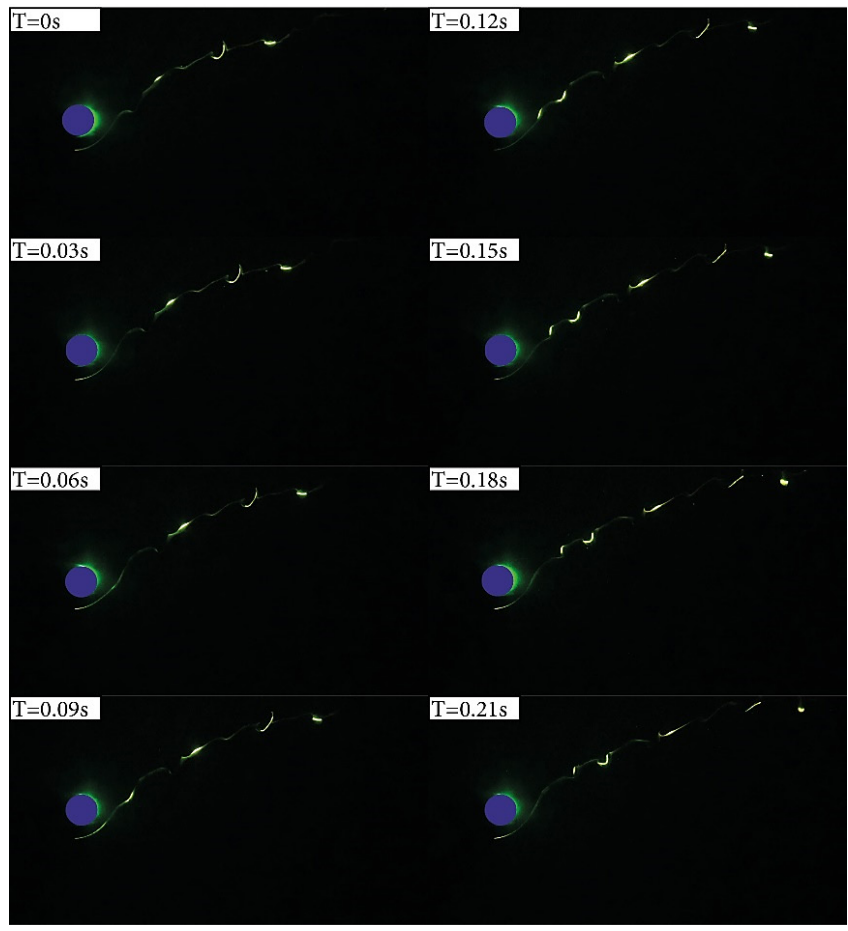

Figure 8. Flow structure formed behind the cylinder at $120 \mathrm{rpm}$ rotation clockwise at $\mathrm{Re}=1000$

\section{CONCLUSIONS}

In this study, flow around a circular cylinder $(D=25 \mathrm{~mm}$ diameter) has been visualized at $\mathrm{Re}=1000$ for different rotational conditions.

- It has been observed that the formation of the Karman vortex street with a certain frequency for the fixed cylinder and its progress in the flow direction. It is observed that the vortices formed by separating the cylinder from the boundary layer progress in time and grow larger as it moves away from the cylinder.
- The clockwise rotation was given one-way rotation speed and it was observed that the wake region, where the boundary layer separation was shifted at high rotation speeds, narrowed towards the cylinder and that the Karman vortex street and Kelvin Helmhotz instability formed in the flow direction did not occur.

- It has been determined that the wake region zone, where the boundary layer separation is shifted at high rotation speeds, narrows towards the cylinder and does not occur in the Karman vortex street and Kelvin Helmhotz instabilities that are formed in the flow direction.

- With the increase of the rotational speed, an acceleration layer occurs between the fluid velocity in the cylinder wake in the flow direction and the flow from the circumference of the cylinder with the increasing speed. This situation caused the vortex ruptures in the flow direction to disappear completely and Kelvin Helmhotz fluctuations to occur.

\section{ACKNOWLEDGEMENT}

This study was supported by the Scientific and Technological Research Council of Turkey (TUBITAK) within the scope of the project named TUBITAK-218M357. Thanks to TUBITAK for their support. We are also very grateful to the reviewers for their valuable comments, which have been utilized to improve the quality of the paper.

\section{REFERENCES}

[1] Manelil, P., N., Tiwari, S. (2019). Wake characteristics of a sphere performing transverse rotary oscillations. European Journal of Mechanics- B/Fluids, 77:148-161, https://doi.org/10.1016/j.euromechflu.2019.04.009

[2] Akar, A., M. (2008). Vortex Control ın Side by Side Circular Cylinders in Shallow Waters. Çukurova University, Institute of Natural and Applied Sciences, Ph.D. Thesis.

[3] Cheng, M. (2007). Characteristics of two-dimensional flow around a rotating circular cylinder near a plane wall. Physics of Fluids 19, 063601, https://doi.org/10.1063/1.2738608

[4] Fallah, K., Fardad, A. A., Fattahi, E., Zadeh, N. S., Ghaderi, A. (2012). Numerical simulation of planar shear flow passing a rotating cylinder at low Reynolds numbers. Acta Mechanica, 223:221-236, DOI 10.1007/s00707-011-0561-4

[5] Guo, X. H., Lin, J. Z., Tu, C. X., \& Wang, H. L. (2009). Flow past two rotating circular cylinders in a side-by-side arrangement. Journal of Hydrodynamics, 21(2), 143-151. DOI: 10.1016/S1001-6058(08)60131-6

[6] Firat, E., Ozkan, G. M., Akilli, H. (2017). PIV measurements in the near wakes of hollow cylinders with holes. Experiments in Fluids, 58(5), 39. DOI 10.1007/s00348-017-2334-x

[7] Gad-el-Hak, M., \& Bushnell, D. M. (1991). Separation control: Review. Journal of Fluids Engineering, 113:5.

[8] Dimotakis, P., Tokumaru, P. T. (1991). Rotary oscillation control of a cylinder wake. J. Fluid Mech. 224:77-90.

[9] Lam, M. K. (2009). Vortex shedding flow behind a slowly rotating circular cylinder. Journal of Fluids and Structures. 25:245-262. https://doi.org/10.1016/j.jluidstructs.2008.04.005 
[10] Sengupta, T. K., Deb K., Talla, S. B. (2007). Control of flow using genetic algorithm for a circular cylinder executing rotary oscillation, Comput. Fluids, 36(3), 578-600, https://doi.org/10.1016/j.compfluid.2006.03.002.

[11] Pralits,J. O., Brandt,L., Giannetti, F. (2010). Instability and sensitivity of the flow around a rotating circular cylinder, J. Fluid Mech., 650: 513-536, DOI: https://doi.org/10.1017/S0022112009993764.

[12] Kumar, S., Lopez,C., Probst,O., Francisco,G., Askari,D., Yang, Y. (2013). Flow past a rotationally oscillating cylinder, J. Fluid Mech., 735:307346. DOI: https://doi.org/10.1017/jfm.2013.469 